\title{
Climbing mechanisms as a central trait to understand the ecology of lianas - a global synthesis.
}

\author{
Arildo Souza Dias ${ }^{1}$, Rafael Oliveira ${ }^{2}$, Fernando Martins ${ }^{3}$, Frans Bongers ${ }^{4}$, Niels Anten ${ }^{5}$, \\ and Frank Sterck ${ }^{4}$ \\ ${ }^{1}$ Goethe-Universität Frankfurt am Main \\ ${ }^{2}$ University of Campinas \\ ${ }^{3}$ Universidade Estadual de Campinas \\ ${ }^{4}$ Wageningen University \& Research \\ ${ }^{5}$ Wageningen University
}

May 3, 2021

\begin{abstract}
Lianas are a distinctive component of tropical forests and their increase in abundance may have profound ramifications for forest composition and ecosystem functioning. However, so far, the current view considers lianas as a single, functional plant type and, therefore, ignores the life history differences among species resulting from their climbing mechanisms. Here, we integrate data from the main functional traits used to characterize plant form and function with global abundance data to reveal that lianas consistently have differences associated with their main climbing mechanisms. Overall, lianas with active climbing were characterized by an acquisitive strategy, showing higher specific leaf area, foliar nitrogen and slightly higher (marginally significant) maximum photosynthetic rates compared to lianas with passive climbing. Using structural equation modeling, we show that across the tropics the abundance of lianas with active climbing is mainly determined by forest structure (stem size distribution). Our study shows that active and passive climbing lianas clearly diverge in their functional traits and factors affecting their distribution. We conclude that a shift in the current view, where lianas are considered a single, functional plant type, is urgently needed to increase our predictability of their effects on tropical forests in the future.
\end{abstract}

\section{Introduction}

Tropical forests contain more than half of the Earth's terrestrial species, store one quarter of the global terrestrial carbon and account for one-third of net primary productivity (Bonan, 2008; Wright, 2010). Therefore, any alteration in the structure or functioning of tropical forests has important consequences for biodiversity conservation, productivity and global carbon budgets. Currently, one of the most eminent changes occurring over the last three decades in tropical forests is the increase in liana abundance and biomass (Schnitzer \& Bongers, 2011; Yorke et al., 2013; Schnitzer, 2018). Lianas (woody climbers) are non-self-supporting plants which rely on trees as supports to grow and to gain access to the forest canopy (Schnitzer \& Bongers, 2002; Gerwing et al., 2006). They are a prominent growth form across lowland tropical forests where they may represent up to $40 \%$ of the woody stems and more than $25 \%$ of the woody species (Gentry, 1991). Lianas invest proportionally more in leaf mass than in stem cross-sectional area than trees, thus, deploying a large canopy of leaves on the host trees that they infest (Putz, 1984; Gerwing \& Farias, 2000). Intense competition for above- and below-ground resources with lianas may limit tree recruitment, growth, reproduction and survival (Putz, 1984; Stevens, 1987; Clark \& Clark, 1990; Schnitzer et al., 2000; Ingwell et al., 2010). Moreover, the negative effect of lianas is greater on late-successional- than on pioneer tree species (Schnitzer \& Carson, 2010); this has enormous consequences for community richness and composition, as well as for 
ecosystem-level dynamics such as carbon storage (Schnitzer \& Bongers, 2011; Schnitzer, 2018). Currently, at least eight studies have provided evidence for increases in liana abundance or biomass, or both, across Neotropical forests (Schnitzer \& Bongers, 2011). However, not all studies support the liana increase hypothesis. For instance, only two studies have evaluated the lianas' growth rates for the African continent and these did not find an increase in their abundance or biomass (Schnitzer \& Bongers, 2011; Schnitzer, 2018, Bongers et al. 2020). One possible explanation for these different results across the tropics may be due to taxonomic and functional trait differences among the species present in each of those continents. In fact, Gallagher \& Leishman (2012), in a global analysis for climbing plants, found a difference in the proportion of species having tendril or twining climbing mechanisms in Africa and the Americas. However, whether these taxonomic differences are related to specific morphological and physiological traits is still not clear. One reason is that most of the studies investigating the morphological and physiological traits in lianas have contrasted them with trees, while few have addressed differences amongst lianas differing in their climbing mechanisms (Carter \& Teramura, 1988; Durigon et al., 2013). Consequently, the current predominant view considers lianas as a uniform group and, so far, no comprehensive work has been carried out to test if different climbing strategies may also account for functional trait variability among liana species (Medina et al., 2021). Recognizing functional and morphological differences among liana climbing mechanisms may be essential for understanding their spatial and temporal patterns of abundance across tropical forests, particularly if the increase in liana abundance and biomass is driven by taxa with a specific climbing mechanism and growth strategy. Therefore, if differences between groups of lianas exist and are not accounted for, this may lead to a misrepresentation of the role of lianas role in forest successional dynamics, which, in turn, will have important implications for our understanding of forest responses to climate change.

Lianas have long been attracting the interest of botanists because of their distinctive climbing mechanisms and growth strategies (Darwin, 1875; Isnard \& Silk, 2009) (see Box 1). The vertical growth of lianas is facilitated via a large diversity of attaching systems (Putz, 1984; Nick \& Rowe, 2004; Isnard \& Silk, 2009; Speck \& Burgert, 2011). Each type of climbing mechanism (see Fig. 1) may differ in carbon allocation and the mechanical support requirements necessary for vertical growth, consequently determining the differences in life history among the liana species. For example, it has been proposed that each type of climbing mechanism determines the maximum distance between supports and that climbing success depends on the maximum stem diameter support that they can colonize (Putz \& Holbrook, 1991; Isnard \& Silk, 2009; Rowe \& Speck, 2004). Darwin, in his book "The climbing movement of plants" (1875) (the first study to investigate the ecological behavior of climbing plants), did not consider species which merely scramble over vegetation without any special organ. He described hook-climbers as having the least efficient climbing mechanism, followed by hook and root climbers, while he considered the more widespread twining and tendril climbers to be the most efficient. Those early observations are in accordance with modern-day measured mechanical properties of wood from different types of climbers. For instance, tendril and twining species generally have more flexible stems, while hook-climbers and scramblers retain relatively stiff stems throughout their lives (Isnard \& Silk, 2009; Speck \& Burgert, 2011; Rowe \& Speck, 2004). Considering the lianas life history, one emerging question is whether an active climbing mechanism, which facilitates easier access to the upper canopy, is associated with a resource-acquisitive strategy via functional traits. The theoretical basis for this hypothesis relies on the fact that the type of climbing mechanism limits the maximum size of the support used for vertical growth (e.g., the stem diameter to which a specific climbing mechanism can attach and, consequently, climb on), as well as the light environment that can be explored (Darwin, 1875; Putz, 1984; Holbrook \& Putz, 1991). Therefore, the success of certain types of climbing mechanisms will differ according to the forest structure and successional stages (Putz \& Chai, 1987; DeWalt et al., 2000). Likewise, there is evidence that the proportion of species with different climbing mechanisms differ significantly between continents (Gallagher and Leishman, 2012), but the main drivers (climate, soil and forest structure) of these patterns are still not explored.

Plant functional types have been used by ecologists to aggregate the enormous number of plant species into a tractable number of functionally similar classes (Ustin \& Gamon, 2010). This classification implies an ecological basis to differentiate between groups of plants responding similarly to changes in their environments 
(Diaz \& Cabido, 1997; Lavorel \& Garnier, 2002), while there is also strong support for significant differences in key traits among plants of different functional types (Wright et al., 2004, 2005; Verheijen et al., 2016); such classification is necessary in order to reduce the need for specific knowledge about each underlying species functional trait. Plant traits are suitable tools for describing the different functional aspects of plants and their relationships to environmental conditions. The various growth and survival strategies of plants in response to abiotic and biotic determinants are reflected by their set of trait values (Westoby et al., 2002; Reich, 2014; Verheijen et al., 2016). An increasing number of global overviews have been reported for various functional groups of plants (e.g., Wright et al., 2005a, b; Poorter et al., 2012; Verheijen et al., 2016) and it becomes clear that several questions characterizing lianas as a group still need to be answered (Wylka et al., 2013; Schnitzer, 2018; Medina et al., 2021). Although the morphology, anatomy and physiology of the climbing organs used by lianas to reach the canopy have been relatively well studied (for a review see Isnard \& Silk, 2009), much less effort has been made to understand if there is a relationship between the type of climbing mechanism and the functional traits related to whole-plant performance, such as the leaf and wood economic spectra traits (Wright et al., 2004; Chave et al., 2009; Reich, 2014). If such links exist, this information would be especially needed to properly account for the role of lianas in forest dynamics via Dynamic Global Vegetation Models (DGVMs) (Verbeeck et al., 2016; Schnitzer et al., 2016, Meunier et al., 2021). Similarly, the few studies which have characterized the drivers of liana abundance and species richness have focused on only a few environmental variables and have mainly compared lianas to trees (Schnitzer, 2005), focused on specific liana clades and biogeographical realms such as the Neotropics (Meyer et al., 2020), or on a particular type of climbing mechanism (Durigon et al., 2013). Therefore, we still do not know how the environment (e.g., climate and soil) and the forest structure drive the proportion of species (richness) and abundance (density) of lianas regarding their main climbing strategies across the large spatial scales.

Classifications of the climbing mechanisms used by lianas may vary substantially throughout literature, ranging from three categories (Vaughn \& Bowling, 2011) to as high as nine in some cases (Addo-Fordjour \& Rahmad, 2015), as recently reviewed by Sperotto et al. (2020). In this study, we followed the recently proposed classification of climbing mechanisms from Sperotto et al. (2020) to establish a framework which considers a continuum from highly-specialized obligate lianas (active climbing) to species with an intermediate behavior (passive climbing) and devoid of highly specialized attachment systems (Fig. 1). Here we analyzed the functional strategies and distribution of lianas, classifying lianas into two main types of climbing mechanisms: active and passive. In general, active climbing comprises twining, tendrils, prehensile branches, twining petioles and prehensile peduncles and inflorescences, while passive climbing comprises scrambling (or clambering, leaning), hooks or grapnels and adhesive roots.

The current perspective regarding the ecology of lianas remains somewhat limited because previous analyses of liana ecology have largely focused on the differences between lianas and trees and, so far, no major synthesis has considered the life history differences among lianas in terms of their climbing mechanisms. Here we synthesize data for lianas regarding the main functional traits used to characterize plant form and function and, thus, classify the liana species according to their main type of climbing mechanisms (Fig. 1, Box 1): active and passive climbing. Furthermore, we integrate the liana species climbing mechanism data with a standardized pantropical plot sampling of the species richness and abundance, in addition to remote sensing data (climate and soil) to explore the following hypotheses:

(1) There is an association between the climbing mechanism and functional traits, therefore, we expect that functional traits differ between active and passive climbing lianas. More specifically, we expect that species with active climbing, that forage vertically through a short-term vigorous growth, show functional traits towards the acquisitive end of the global trait spectra.

(2) The magnitude and direction of the associations between the main drivers (climate, soil and forest structure) of the relative abundance and richness of lianas differ between active and passive climbing species (Table 1). We expect that taller forests will favor active climbers, whereas shorter forests will favor passive climbers. We expect that climate and soil fertility show a positive relationship to species richness for both active and passive climbing, while abundance may respond differently to those variables because recruitment 
is also affected by forest structure (availability of suitable supports).

Here, we first show that functional trait differences are significant between the two main categories of liana climbing mechanisms: active and passive climbing (Sperotto et al., 2020). Subsequently, we show that the factors driving abundance and species richness of lianas across tropical forests differ significantly between active and passive climbing species. Finally, we discuss the need of reconsidering the view of lianas as a single, plant functional type, given the importance of the climbing mechanism to the life history and ecology of lianas.

\section{Box 1. The definition of climbing mechanisms and their importance in the life-history of liana.}

Lianas are a particular type of climbing plant that develop woody stems and have a non-self-supporting habit so they rely upon an external support for vertical growth towards the canopy (Rowe and Speck, 2004; Schnitzer \& Bongers, 2002). In general, the ontogeny of lianas is marked by two distinct phases: firstly, the erect phase, where, after germinating, they can grow vertically adopting a self-supporting habit and can reach up to $2 \mathrm{~m}$ height without the need for an external support and, secondly, the climbing phase (Putz \& Holbrook, 1991), which is marked by a rapid increase in the internode distance with low investment in ramification and foliar expansion until a suitable support is found (Hegarty, 1991; Isnard \& Silk, 2009; Ichihashi and Tateno, 2015). This marked plasticity in response to the availability of support is associated with several changes in the anatomy, morphology and physiology of the leaves, stems and branches; this is a key feature in the life history of many liana species (Putz, 1984; Gentry, 1991; Isnard \& Silk, 2009; Chen et al., 2014). The need for finding a support for vertical growth is also an important determinant of the spatial distribution of lianas inside forests (Putz, 1984; Putz \& Holbrook, 1991; Schnitzer \& Bongers, 2002). For example, tendril species are restricted to attach to trees with smaller diameters than twiner species (Putz, 1984; Putz and Chai, 1987), therefore, in forests with high rates of fragmentation or gap formation, where more small trees are present, tendril species tend to show higher abundance (DeWalt et al., 2005; Letcher \& Chazdon, 2009).

The transition between the self- and non-self-supporting phase described above is also heavily dependent on the type of climbing mechanism that lianas can use to attach to the support structures. Lianas exhibit a diversity of morphological and anatomical variation (Putz \& Holbrook, 1991; Isnard \& Silk, 2009; Rowe \& Speck, 2004) related to the type of attachment and the organs (e.g., leaf, branch or stem) used to adhere to the support structures (climbing strategy). In part, this diversity results from the unique demand to maintain stems that are both flexible to twist, but also strong enough to climb and span across different hosts without breaking during the search for light (Rowe \& Speck, 2004; Isnard \& Silk, 2009).

Species with an active climbing mechanism display a support-searching behavior such as circumnutation, an endogenous growth-related rhythmic movement in which leader shoots (i.e., shoots produced to search for supports) sweep through the air in arcs (Darwin, 1875; Hegarty, 1991; Carlquist, 1991; Putz et al., 1991; Isnard \& Silk, 2009). This greatly increases the possibility of the plant finding suitable supports. On the other hand, species with a passive climbing mechanism do not actively search for support, rather, they merely grow over the host plants without any searching movements such as circumnutation. Additionally, species with a passive climbing mechanism may have adhesive roots or specialized grapnels or hooks to ensure their attachment to supports.

\section{Material and Methods}

Assembling a global dataset of liana functional traits and climbing strategies

We built a dataset from peer-reviewed studies and published databases, searching for studies that measure functional traits considered important to explain plant form and function along a continuum of fast acquisitive to slow, conservative species (Reich, 2014; Diaz et al., 2015) in liana species. We restricted our analyses to traits having the most information available at the species level: individual leaf area (LA), specific leaf area (SLA), maximum leaf photosynthetic capacity per area $\left(\mathrm{A}_{\max }\right)$, nitrogen foliar content per mass $\left(\mathrm{N}_{\text {mass }}\right)$, wood density -(WD) and seed mass -(SM). We focused on angiosperm woody species (lianas) as the traits 
analyzed may vary significantly between woody and herbaceous species. Palm climbers and monocot species, such as from the genera Smilax spp., were not included in the analyses given that monocots have a different ecophysiological behavior. In our study we adopted the liana definition as proposed by Gerwing et al. (2006): "climbing plants that produce true wood (xylem tissues derived from a vascular cambium) and that germinate on the ground but lose their ability to support themselves as they grow, so they have to rely on external physical support to ascend to the canopy". Therefore, we did not include species with a classification of "hemiepiphyte". However, we noticed that the criterion of woodiness would exclude some genera of dicotyledons, such as Passiflora, Ipomea and many Cucurbitaceae, which have perennial fibrous stems but are abundant and important in many liana inventories (Gentry, 1991; Gerwing et al., 2006). Thus, we have also included those genera and respective families in our analyses. Species present in more than one study and showing different classifications for their climbing strategies were excluded to avoid uncertainties about their true climbing mechanisms. If the liana climbing mechanism was not informed in the original studies, we searched this information through a thorough literature survey in the literature. We checked online sources and databases (e.g., virtual herbaria, online floras) to ascribe the climbing mechanism to all liana species we used in the analyses (Appendix S1). We classified liana climbing mechanisms according to the classification proposed by Sperotto et al. (2020) and we focused on the comparison between the active and passive climbing mechanisms used by lianas (see Box 1 for a complete explanation, Fig. 1 and Appendix $\mathrm{S} 1$ in Support Information). We only included species identified at the binomial level, to facilitate the name standardization of the species among different datasets. Species names were matched against accepted names in The Plant List using the "taxonstand" R package (Cayuela et al., 2012). We also limited our search to studies reporting the traits of adult plants growing in natural conditions and, therefore, we did not include assembled data for seedlings and saplings, in addition to plants growing in experimental conditions. In total, we assembled data from 42 studies encompassing 755 species of lianas, with information for at least one of the above traits for each species (Appendix S1).

Extracting data for species richness and abundance, forest structure, climate and soil

\section{Species abundance and richness}

We used as data for the abundance and basal area of liana species from the Gentry's transects, accessed through the BIEN Database (R package "BIEN" version 4.1.1, Maitner et al., 2017). We restricted our analyses to plots only across the tropics, using the same criteria as Schnitzer (2005). In summary, we restricted the data set to forests that were classified as tropical lowland dry, moist or wet forests (according to Holdridge, 1967) and which had [?] $500 \mathrm{~mm}$ annual precipitation and were [?] 1,000 $\mathrm{m}$ above sea level to reduce the confounding effects of latitude, elevation and temperature among the sites. Abundance was defined as the total number of individuals and richness as the total number of species, per plot, for each type of climbing mechanism (active and passive). All plot data comes from the Gentry's transects, openly available through the BIEN Database. We chose these data because they are standardized and represent one of the most comprehensive available inventory data on the liana species richness and abundance across the tropics.

\section{Environmental and forest structure variables}

We used forest structure, climate and soil variables as predictors of liana abundance (Figure S1). Canopy height, as derived from spaceborne LiDAR, has been used as a proxy for forest height (Lefsky, 2010; Simard et al., 2011) and has also been used in macroecological analyses to approximate the amount of vertical habitat and niche space, for example, of climbing palms (Couvreur et al., 2015), lianas (Meyer et al., 2020) and primates (Gouveia et al., 2014). We used the 1-km resolution canopy height map from Simard et al. (2011) and extracted the mean canopy height for each point location correspondent to the plots with information for the abundance of the lianas (Gentry's transects). We calculated the gap phase index (GP) based on the relative size distribution of stems per each 0.1 ha plot following Feeley et al. (2007). GP was calculated as follows: $\mathrm{GP}=\ln \left(\left[\mathrm{BA}_{30}+1\right] /\left[\mathrm{BA}_{10}+1\right]\right)$, where $\mathrm{BA}_{30}$ is the total basal area of all stems with dbh [?] $30 \mathrm{~cm}$ (dbh - diameter at $1.30 \mathrm{~m}$ above ground) and $\mathrm{BA}_{10}$ is the total basal area of all stems with dbh

[?] $2.5 \mathrm{~cm}$ and [?] $10 \mathrm{~cm}$ (Feeley et al., 2007). For these calculations we considered only the stems of trees 
and shrubs that could provide more suitable support for the lianas to climb on and which would not include other growth forms such as hemiepiphytes or herbs, which were also sampled in the methodology used by Gentry.

As bioclimatic variables may be highly colinear with each other and as we were primarily interested in the influence of specific climatic variables, per se, we quantified the influence of climate on the abundance and richness of the liana climbing mechanisms by extracting the mean annual precipitation (in millimeters per year) and mean annual temperature (in degrees Celsius) for each plot location from the 30 arcsec resolution CHELSA dataset (Krager et al., 2007). We also included the climatic water deficit, a proxy for the monthly water deficit experienced by plants (Aragão et al., 2007). For edaphic conditions, we extracted the cation exchange capacity (CEC) (a general proxy for soil fertility; cmol $(+) \mathrm{kg}^{-1}$ ) at $250 \mathrm{~m}$ resolution from the SoilGrids website (https://soilg rids.org/).

\section{Statistical analysis}

We assessed the differences in the functional traits between active and passive climbing species by using t-tests. We $\log _{10}$-transformed the leaf area and seed mass before the analysis to meet the requirements of normality checked by quantile plots. In addition, we quantified the phylogenetic signal that indicates to what extent phenotypic expression is explained by the lineage to which a species belongs. We quantified the phylogenetic signal using the Blomberg's $K$ (Blomberg, 2003). We first generated a phylogenetic tree including all liana species for which we have functional trait information using the V.PhyloMaker R package (Jin \& Qian, 2019). Consequently, we pruned separated phylogenies, one for each of the two main climbing mechanisms, using the original tree with all species as a base phylogeny. Subsequently, we pruned a tree for each group-trait combination independently, removing taxa for which information trait information was not available. Values of $K=1$ imply that a trait shows exactly the amount of phylogenetic signal expected under a null, stochastic model of character evolution (Brownian motion evolution) (Blomberg, 2003). If $K$ does not differ from zero, then the trait has no phylogenetic signal. The statistical significance of $K$ was assessed via permutation tests with 1000 randomizations. The significance of the phylogenetic signal was based on the variance of phylogenetically independent contrasts relative to tip shuffling randomization implemented by the phylosignal function of thepicante package in $\mathrm{R}$ (Freckleton et al., 2002). The P-values were determined by comparing the variance of the standardized independent contrasts for the tip values against the randomized data.

We used piecewise structural equation modeling to examine the hypothesized relationships between abundance and richness per climbing mechanism and the climate, soil and forest structure variables (Lefcheck, 2016). One of the main advantages of the structural equation models (SEM) is that it allows partitioning the total effect of the relationship between variables into direct and indirect effects (Grace, 2006). We evaluated the generality of the model across the tropics by controlling the effect of biogeography (continent). We built the theoretical SEM (Appendix S2, Fig. S1) based on hypothesized and established relationships between environmental variables and the abundance and species richness of lianas and plants in general (Table 1). We also evaluated if the ratio of active, relative to passive, climbing species richness differed among biogeographic realms using a generalized linear model. All analyses were performed in the $\mathrm{R}$ environment (R Core Team 2018).

\section{Results}

\section{Trait variation explained by climbing mechanisms}

We found functional traits for 755 liana species: 551 representing active climbing species and 204 representing passive climbing species. Species employing an active climbing mechanism were characterized by traits associated with acquisitive strategy compared to species with a passive climbing mechanism (Fig. 2). Active climbing species showed higher specific leaf area (t-test, $P=0.0001$; active $=187$, passive $=57$ ), larger total leaf area (t-test, $P=1.7 \mathrm{e}-18$; active $=380$, passive $=136)$, higher leaf nitrogen content $(\mathrm{t}$-test, $P=$ 0.02 ; active $=129$, passive $=38)$ and slightly higher maximum photosynthetic rate per mass unit (t-test, $P=0.05$; active $=56$, passive $=20)$ compared to passive climbing species (Fig. 2a-d). We did not find 
differences in wood density between the active and passive climbing species (t-test, $P=0.2$; active $=119$, passive $=19)($ Fig. 2e). Seed mass was higher in the active compared to the passive climbing species (t-test, $\mathrm{P}=0.0006$; active $=225$, passive $=69)($ Fig. $2 \mathrm{f})$. Overall, only the passive climbing species showed a significant phylogenetic signal (Blomberg's $K$ ) considering the functional traits we analyzed here (Appendix S2, Table S2). Passive climbing species showed significant phylogenetic signals for SLA, LA, $\mathrm{N}_{\text {mass }}$ and seed mass. These results indicate that active climbing species tended to show patterns of trait variation among species that were independent of phylogenetic relatedness.

\section{Determinants of abundance and species richness among lianas differing in climbing mechanisms}

Across all 122 plots, lianas accounted for a total of 4500 individual stems ([?] $2.5 \mathrm{~cm}$ dbh). Lianas with an active climbing mechanism accounted for 3686 individual stems, belonging to 592 species and 194 genera. The remaining 814 individual stems represented lianas with a passive climbing mechanism, belonging to 170 species and 86 genera. The final multilevel model (structural equation model), including the density and species richness (Fig. 3,4) for both active and passive climbing species, was very well supported by the data (Fisher's $\mathrm{C}=0.396$; d.f. $=2 ; P=0.82$ ) (Table S3). As the SEM results considering the basal area of active and passive climbing species were qualitatively similar to the SEM results for abundance (Appendix S2, Fig. S2), we refer only to the abundance and species richness results in the main text.

The results of our SEM models revealed a clear effect of forest structure and climate on the abundance and richness of active and passive climbing species, after controlling for the biogeographic region (Fig. 3). As the results of the SEM models including the climatic water deficit were similar to those including the mean annual precipitation (Table S3), we refer (in the main text) only to the results including the mean annual precipitation. The gap forest index (stem size distribution) had a positive effect on the abundance and richness of active species, but only on the richness of passive climbing species (Fig. $3 \mathrm{a}, \mathrm{b}$ ). Canopy height, another metric of forest structure, had a significant negative effect only on the richness of the passive climbing species (Fig. $3 \mathrm{~b}$ ). Among the climate variables, temperature was the strongest predictor of abundance and richness (marginally significant) of the active climbing species, with a greater abundance and richness of active climbing species being associated with higher temperatures (Fig. 3, 4). The temperature did not have a significant effect on either the abundance or richness of passive climbing species, while precipitation was positively associated with the richness of passive climbing species (Fig. 4 b). Soil fertility (CEC) was negatively associated with the richness of both active and passive climbing species, but not with the abundance of both active and passive climbing species (Fig. $4 \mathrm{a}, \mathrm{b}$ ). We found no significant indirect effects, via the canopy height, of the climate (temperature and precipitation) and soil fertility (CEC) on the abundance of either active or passive climbing species, nor was there such an effect on the richness of the active climbing species, however, soil fertility, temperature and precipitation did have an indirect effect on the richness of passive climbing species (Fig. $4 \mathrm{~b}$ ).

\section{Discussion}

Our study demonstrates a strong functional divergence associated with the liana climbing mechanism. Specifically, our results reveal that species with an active climbing mechanism are characterized by traits associated with an acquisitive strategy compared to species with a passive climbing mechanism. We also find that across the tropics the abundance of active climbing species is best explained by temperature and forest structure (the tree size distribution), while these factors were poor predictors of the abundance of passive climbing species. Collectively, our results indicate that, when accounting for their climbing mechanism, lianas clearly diverge in terms of functional strategies and the environmental factors that affect their distribution. This highlights the need to reconsider the current view of treating lianas as a single, functional plant type and may have important implications for the understanding and prediction of the effects of lianas on tropical forests in the future.

Trait variation explains the differences between climbing mechanisms of lianas

Most of the current knowledge of liana functional traits comes from studies that compare lianas to tree species (reviewed in Wyka et al., 2015; Schintzer, 2018). These studies showed that, compared to trees, 
lianas specialize toward the acquisitive end of the global trait spectra, although there remains a huge trait variability within lianas. Differently from previous studies, we showed that a significant part of this trait variability can be explained by the climbing mechanism of lianas. We found that, compared to passive climbing species, the active climbing species showed higher values of leaf economic traits (SLA, $\mathrm{N}_{\text {mass }}$ and $\left.A_{\max }\right)$ that are typically associated with an acquisitive strategy. The maximal photosynthetic rate per leaf area $\left(\mathrm{A}_{\max }\right)$, the amount of leaf area per unit leaf mass (SLA) and the amount of nitrogen per leaf content $\left(\mathrm{N}_{\text {mass }}\right)$ are all related to several important functional traits, such as leaf longevity, which, in turn, influence performance and help to account for the differences in the species growth and survival across gradients (Wright et al., 2004; Sterck et al., 2011; Laughlin et al., 2020). The growth and potential acclimation of lianas, inside and across forests, depends on their climbing mechanism (Putz, 1984; DeWalt et al., 2005; Putz \& Holbrook, 1991), however, in contrast to trees, it is the rate of stem elongation, more than the investment in diameter increase, that plays a central role in the liana's ability to find suitable support structures and successful colonization. For instance, there is evidence that lianas exhibit higher rates of height growth compared to trees (Schnitzer, 2005) and that AC species have higher rates of shoot elongation compared to PC species (Teramura et al., 1991). Although this evidence is still limited, the clear differences in the economic traits that we found between the $\mathrm{AC}$ and $\mathrm{PC}$ species support the idea that the liana vertical growth strategy may be determined by the type of climbing mechanism.

Wood density did not differ between the AC and PC species suggesting that the stem construction costs are widely constrained across lianas, irrespective of their climbing mechanism (Fig. 2). One reason that wood density poorly represents differences across lianas species may be due to the limited investment in wood construction (low biomechanical demand) and the resulting constant replacement of old branches in order to keep a high photosynthetic to non-photosynthetic organ ratio. This idea of rapid branch and stem turnover rates in lianas is supported by previous studies showing that lianas grow towards the canopy by discharging a large number of branches (Ichihashi et al., 2010; Ichihashi \& Tateno, 2011, 2015), whereas, in comparison, trees grow higher by accumulating supporting tissue in the stems to stand upright. For instance, Ichihashi \& Tateno (2015) found that the stem turnover varied according to the liana's climbing mechanism, with both stem extension and relative stem losses being the greatest in the twiner species (Actinidia arguta and Celastrus orbiculatus) and the least in a root climber species (Schizophragma hydrangeoides), probably reflecting the different demands for finding well-lit sites and new hosts (Selaya and Anten, 2008).

We found that active climbing species have higher values of size-related traits, specifically seed mass and leaf area, when compared to passive climbing species. In general, large stature plants tend to have large leaves and large, heavy diaspores (Diaz et al., 2015). Seed mass is also a trait associated with larger seedling size and, therefore, higher changes of recruitment (Moles et al., 2005; Gilbert et al., 2016). Data related to liana allometry are limited, but there is evidence that active climbing species tend to reach the canopy and spread among different host crowns, developing an aggressive strategy in terms of growth and space occupation compared to passive climbing species (Ichihashi \& Tateno, 2015). This suggests that the larger leaves and seed mass we found in the active climbing species could be a result of their faster stem lengthening and growth strategy.

Overall, we found strong evidence for the idea that active climbing species, compared to passive climbing species, specialize towards a fast strategy (i.e., rates of resource acquisition and processing) along the plant global trait spectra (Reich, 2014; Diaz et al., 2015). The fact that active climbing species do not show a phylogenetic signal considering the traits analyzed here, suggests that the acquisitive strategy of active climbing species results from a convergence ecological strategy. This clear distinction between active climbing and passive climbing species is contrary to the current view considering lianas as a uniform, plant functional type and, instead, supports the hypothesis of niche differentiation among liana species related to the type of climbing mechanism (Icischi \&Tateno, 2011; Wyka et al., 2014).

Drivers of variation in abundance and species richness between climbing mechanisms across the tropics

Our results showed that, across the tropics, climate and forest structure are the main factors controlling liana abundance and species richness, with both factors having different effects and explanatory powers depending 
on the type of climbing mechanism used by the lianas.

The forest gap index was positively related to the abundance of active climbing species but not to the abundance of passive climbing species, while, contrary to our expectation, canopy height was not significantly related to either active or passive climbing abundance. The influence of the forest structure on liana recruitment largely depends on the climbing mechanisms used by lianas to ascend the canopy. For instance, the host trunk diameter restricts mainly tendril and twiner species (active climbing in Fig. 1), but variation in host size (as indicated by the forest gap index) within a forest may allow them to climb from one tree to the next and, thus, reach the forest canopy. Hooks and scramblers (passive climbing in Fig. 1), in general, do not reach the upper parts of the canopy (Rowe \& Speck, 2004; Sandrine \& Isnard, 2009), particularly because these species lean on their host and cannot easily climb beyond the host's height to reach the canopy, while root climbers can attach to any size of trunk but cannot cross between different trunks. It is clear, therefore, that the host size and the climbing behavior of the liana determines the differences in the potential environment that lianas can explore within forests (Ichihashi \& Tateno, 2011, 2015). This variation suggests a niche differentiation among lianas; this could be an important mechanism underlying the diversification and coexistence of lianas species across tropical forests.

The species richness of active and passive climbing were both positively associated with the forest gap index, but only the species richness of the passive climbers was negatively related to canopy height. Our results, in part, contrast with a recent study showing that across the Neotropics the liana species richness from the tribe Bignoniae, which in general have a type of active climbing mechanism (tendril-climbers), was positively related to canopy height (Meyer et al., 2020). There are two possible explanations for these differences. Firstly, our study analyzed a wide group of liana species and not only a specific clade and, secondly, our study focused on community plot-level data, while Meyer et al. (2020) focused on grid cells. It is possible that at the plot-level scale, successional status and disturbance, as indicated by the forest gap index, might represent a stronger filter to active climbing species than canopy height by providing more light and suitable supports. Indeed, disturbance and forest structure have been shown to play an important role in the maintenance of liana diversity and density in forests (Schnitzer \& Carson, 2000; DeWalt et al., 2000; Schintzer \& Bongers, 2002; van der Heijden \& Phillips, 2008, 2009). On the other hand, the richness of the passive climbing species in the present study was found to be negatively related to canopy height. It is possible that the passive climbing species have less opportunity to grow towards regions of high light intensity in a tall forest canopy, whereas the active climbing species can forage more effectively and, thus, face no such loss in richness in relation to canopy height.

In our study, the temperature was positively related to the abundance and richness of active climbing species, while precipitation had a positive effect only on the richness of the passive climbing species. These results are broadly consistent with previous studies considering lianas in general (Couvreur et al., 2015; DeWalt et al., 2015; van der Heijden \& Phillips, 2009), however, our study revealed that climate contributes differently to explain the abundance and richness of lianas when accounting for the climbing mechanism. The fact that only precipitation was strongly positively related to the passive climbing species richness supports previous results which showed that root climbers (which have a passive climbing mechanism) correlate positively with rainfall across the globe (Durigon et al., 2013). The positive relationship between active climbing abundance and species richness with temperature may also reflect the higher potential of acclimation and phenotypic plasticity of active climbing species. For instance, Carter \& Teramura (1991) analyzed the photosynthetic acclimation of lianas differing in climbing mechanisms and found that adhesive discs displayed greater physiological acclimation to low light environments, while tendril climbers were found to have the broadest physiological plasticity to high light environments. Overall, the positive relationship between the forest gap index (significant) and temperature (marginally significant) with the active climbing species richness are compatible with our functional trait analyses of showing that active climbing species have an acquisitive strategy.

The soil variables, at least at the macroecological scale, that were analyzed in the present study were only weakly associated with the abundance of the active and passive climbing species, this is consistent with the 
soil properties usually being more important predictors of liana distribution at the fine spatial scale (Powers, 2015; van der Heijden \& Phillips, 2009). The richness of both active and passive climbing species generally decreased with soil fertility (i.e., with increasing CEC), which was in contrast to our initial expectation that higher nutrient availability should increase liana species richness (Gillman et al., 2015; Pausas \& Austin, 2001). This, in part, may be due to there being further differences in the soil parameters between regions that were unaccounted for in our analysis.

We found that the relative richness of active to passive climbing species was significantly higher only in the Neotropics compared to the other biogeographical realms (Appendix S2 - Table S1, Fig. 4). One possible explanation might be related to differences in canopy height and forest history, leading to a strict association between liana ecology and 3D forest structure in some biomes and continents, but not others. For instance, differences in the vegetation structure may promote specific climbing mechanisms (e.g., tendrils) (Lohmann, 2003) and, thus, favors the evolution of alternative lifeforms across these biogeographical realms (e.g., Neotropics versus Afrotropic). In fact, Couvreur et al. (2015) showed a link between the diversification of the climbing life-form in palms and the forest canopy height. Likewise, ancestral area reconstructions suggest that almost all ancestors of the currently recognized genera of the tribe Bignonieae, where most of the species are tendril climbers, inhabited forest habitats (Lohmann et al., 2013). Additional work integrating abundance patterns with a comprehensive species-level phylogeny of lianas would be important to clarify the link between ecology and the evolution of the climbing mechanism.

\section{Summary and Future Directions}

We provide strong evidence that the type of climbing mechanism is a central characteristic to understand the functional ecology and distribution of lianas. Our synthesis is the first to jointly assess the global-scale patterns of distribution and functional trait variations of lianas while accounting for their type of climbing mechanism.

It has been shown that the increase in lianas abundance and biomass in recent decades across the tropics is not uniform (Schnitzer \& Bongers, 2011). An increase is evident in the Neotropics, while there has been no increase in the Afrotropic and mixed results for Indo-Malay (Schnitzer \& Bongers, 2011; Schnitzer, 2018). Our results suggest that these differences might be related to a combination of functional trait differences and factors affecting the distribution among liana species that are related to the climbing mechanisms and growth strategies of lianas. Furthermore, we showed that the relative richness of lianas with active climbing compared to passive climbing differs significantly between the Neotropics and other biogeographical realms (Fig. 4), suggesting that the consistent increase in lianas abundance and biomass across the Neotropics (Schnitzer and Bongers, 2011), compared to other biogeographic regions, could be a result of the preponderance of active climbing species in this region. Future studies should attempt to test this hypothesis, ideally using demographic data and information about the liana climbing mechanisms from different biogeographic regions. Increases in temperature and in forest disturbance (leading to increasing light availability and trees with thinner trunks) are likely to act synergistically to increase the abundance of lianas, particularly species with an aggressive strategy such as the active climbing species whose abundance strongly depends on temperature and forest structure. We suggest that future studies should use demographic data from local and large spatial scales, combined with data on the liana climbing mechanisms and functional traits to test the generality of our findings.

Our finding that there is a biogeographic contingency in the abundance of lianas according to their main type of climbing mechanism also has important implications for modeling the effect of lianas on the forest structure at a large spatial scale. For instance, the first DGVM to include lianas considered them as a single, plant functional type (di Porcia e Brugnera et al., 2020). The implicit assumption is that there is no, or very little, functional variation within the lianas as a group. However, our results show that there is significant functional variation among lianas and that an important part of this variation can be explained by the type of climbing mechanism. The inclusion of lianas in DGVMs is definitely an important step for increasing our ability to understand their effects on forest dynamics, however, our results suggest that representation of lianas as a single, plant functional type in DGVMs may be premature and deserves more investigation in 
the future.

\section{Conclusions}

The evolution of climbing habit and its implication for the life history of lianas is still an understudied topic in ecology, but this information is essential to link a species response to climate change across multiple scales. As we showed in this study, when accounting for the climbing mechanism, lianas clearly diverge in their functional traits and abiotic factors which drive their distribution. We argue that in order to understand the main causes of the increases in liana abundance and biomass that are being observed in tropical forests, it will be crucial to gather data at the species-, population- and plot-levels across forests and to analyze them, taking into account their life-history differences such as the type of climbing mechanism. As these data continue to be generated and become available through databases, such as BIEN and TRY in recent years, we shall be able to understand better the ecology of lianas and their role in tropical forest dynamics.

\section{Acknowledgements}

The first author gratefully acknowledges the Sao Paulo Research Foundation (FAPESP, grant number 2010/11459) and the National Council for Scientific and Technological Development (CNPq - 233206/2014-0) for financial support. FRM thanks CNPq for the productivity grants (process code 306110/2019-9). The first author also thanks Somayeh Shadkam for providing useful comments on the first draft of the manuscript. We declare no conflict of interest. We thank all the contributors for the invaluable data provided to the BIEN database (http://bien.nceas.ucsb.edu/bien/people/data-contributors/).

\section{References}

Addo-Fordjour, P. \& Rahmad, Z.B. (2015). Patterns of liana abundance, reproductive traits and liana-tree relationships in relation to forest management in a tropical forest in Ghana. J. Sustain. For., 34, 832-857.

Aragao, L.E., Malhi, Y., Roman-Cuesta, R.M., Saatchi, S., Anderson, L.O. \& Shimabukuro, Y.E. 2007. Spatial patterns and fire response of recent Amazonian droughts. Geophysical Research Letters , 34: L07701.

Bongers, F., Ewango, C.E., van der Sande, M.T. and Poorter, L. (2020). Liana species decline in Congo basin contrasts with global patterns.Ecology, 101, p.e03004.

Blomberg, S.P., Garland, T., Ives, A.R., Lomberg, S.I.P.B. \& Arland, T.H.G. (2003). Testing for phylogenetic signal in comparative data: behavioral traits are more labile. Evolution , 57, 717-45.

Bonan, G.B. (2008). Forests and climate change: forcings, feedbacks, and the climate benefits of forests. Science, 320, 1444-1449.

Carlquist, S. (1991). Anatomy of vine and liana stem. In: The Biology of Vines, ed(s). Putz, F.E. \& Mooney, H.A. Cambridge University Press, Cambridge, UK, pp. 53-71.

Carter, G.A., Teramura, A.H., Journal, A. \& Jul, N. (1988). Vine photosynthesis and relationships to climbing mechanics in a forest understory. Am. J. Bot. , 75, 1011-1018.

Cayuela, L., Granzow-de la Cerda, I., Albuquerque, F.S. \& Golicher, D.J. (2012). Taxonstand: An r package for species names standardisation in vegetation databases. Methods Ecol. Evol ., 3, 1078-1083.

Chave, J., Coomes, D., Jansen, S., Lewis, S.L., Swenson, N.G. \& Zanne, E. A. (2009). Towards a worldwide wood economics spectrum. Ecol. Lett. , 12, 351-366.

Chen, Y.-J.J., Bongers, F., Zhang, J.-L.L., Liu, J.-Y.Y. \& Cao, K.-F. (2014). Different biomechanical design and ecophysiological strategies in juveniles of two liana species with contrasting growth habit.Am. J. Bot. , 101, 925-934.

Clark, D.B. \& Clark, D.A. (1990) Distribution and effects on tree growth of lianas and woody hemiepiphytes in a Costa Rican Tropical Wet Forest. J.Trop. Ecol. , 6, 321-331. 
Couvreur, T.L., Kissling, W.D., Condamine, F.L., Svenning, J.C., Rowe, N.P. \& Baker, W.J. (2015). Global diversification of a tropical plant growth form: Environmental correlates and historical contingencies in climbing palms. Frontiers in Genetics , 5, 1-18. https://doi.org/10.3389/fgene.2014.00452

Darwin, C. 1875. The movements and habits of climbing plants. John Murray, London, UK.

Dewalt, S.J., Schnitzer, S.A. \& Denslow, J.S. (2000). Density and diversity of lianas along a chronosequence in a central Panamanian lowland forest. J. Trop. Ecol. , 16, 1-19.

di Porcia e Brugnera, M., Fischer, R., Taubert, F., Huth, A. \& Verbeeck, H. (2020). Lianas in silico, ecological insights from a model of structural parasitism. Ecol. Modell. , 431, 109159.

Diaz, S. \& Cabido, M. (1997). Plant functional types and ecosystem function in relation to global change. J. Veg. Sci. , 8, 463-474.

Diaz, S., Kattge, J., Cornelissen, J.H.C., Wright, I.J., Lavorel, S., Dray, S., et al. (2015). The global spectrum of plant form and function. Nature, 529, 167-171.

Durigon, J., Duran, S.M. \& Gianoli, E. (2013). Global distribution of root climbers is positively associated with precipitation and negatively associated with seasonality. J. Trop. Ecol. , 29, 357-360.

Feeley, K.J., Avies, S.T.J.D., Erez, R.O.P. \& Ubbell, S.T.P.H. (2011). Directional changes in the species composition of a tropical forest.Ecology, 92, 871-882.

Gallagher, R. V. \& Leishman, M.R. (2012). A global analysis of trait variation and evolution in climbing plants. J. Biogeogr. , 39, 1757-1771.

Gentry, A. H. (1991). The distribution and evolution of climbing plants. In: The Biology of Vines, ed(s). Putz, F.E. \& Mooney, H.A. Cambridge University Press, Cambridge, UK, pp. 3-42.

Gerwing, J.J., Schnitzer, S.A., Burnham, R.J., Bongers, F., Chave, J., DeWalt, S.J., Ewango, C.E.N., Foster, R., Kenfack, D., Martinez-Ramos, M., Parren, M., Parthasarathy, M., Perez-Salicrup, D.R., Putz, E.F. \& Thomas, D.W. 2006. A standard protocol of liana censuses.Biotropica, 38, 256-261.

Gillman, L.N., Wright, S.D., Cusens, J., McBride, P.D., Malhi, Y. \& Whittaker, R.J. (2015). Latitude, productivity and species richness. Glob. Ecol. Biogeogr. , 24, 107-117. https ://doi. org/10.1111/geb.12245

Gouveia, S.F., Villalobos, F., Dobrovolski, R., Beltrao-Mendes, R. \& Ferrari, S.F. (2014). Forest structure drives global diversity of primates. J. Anim. Ecol. , 83, 1523-1530.

Hegarty, E.E. (1991). Vine-host interactions. In: The Biology of Vines, ed(s). Putz, F.E. \& Mooney, H.A. Cambridge University Press, Cambridge, UK, pp. 347-375.

Ichihashi, R. \& Tateno, M. (2011). Strategies to balance between light acquisition and the risk of falls of four temperate liana species: to overtop host canopies or not? J. Ecol. , 99, 1071-1080.

Ichihashi, R. \& Tateno, M. (2015). Biomass allocation and long-term growth patterns of temperate lianas in comparison with trees. New Phytol. 207, 604-612.

Iida, Y., Poorter, L., Sterck, F.J., Kassim, A.R., Kubo, T., Potts, M.D., et al. (2012). Wood density explains architectural differentiation across 145 co-occurring tropical tree species.Funct. Ecol. , 26, 274-282.

Ilbert, B.E.G., Right, S.J.O.W., Andau, H.E.C.M.U., Itajima, K.A.K., Gilbert, B., Al, E.T., et al. (2006). Life history trade-offs in tropical trees and lianas. Ecology , 87, 1281-1288.

Ingwell, L.L., Wright, S.J., Becklund, K.K., Hubbell, S.P., Schnitzer, S.A., Joseph Wright, S., et al. (2010). The impact of lianas on 10 years of tree growth and mortality on Barro Colorado Island, Panama.J. Ecol. , 98, 879-887.

Isnard, S. \& Silk, W.K. (2009). Moving with climbing plants from Charles Darwin's time into the 21st century. Am. J. Bot. , 96, 1205-1221. 
Jin, Y. \& Hong, Q. (2019). V.PhyloMaker: an R package that can generate very large phylogenies for vascular plants. Ecography, 42, 1353-1359.

Laughlin, D.C., Gremer, J.R., Adler, P.B., Mitchell, R.M. \& Moore, M.M. (2020). The net effect of functional traits on fitness. Trends Ecol. Evol. , 35, 1037-1047.

Lefcheck, J.S. (2016). piecewise SEM: Piecewise structural equation modelling in $\mathrm{r}$ for ecology, evolution, and systematics. Methods Ecol. Evol. , 7, 573-579.

Lefsky, M.A. (2010). A global forest canopy height map from the Moderate Resolution Imaging Spectroradiometer and the Geoscience Laser Altimeter System. Geophysical Research Letters, 37, L15401. https :// doi.org/10.1029/2010G L043622

Letcher, S.G. \& Chazdon, R.L. (2009). Lianas and self-supporting plants during tropical forest succession. For. Ecol. Manage. , 257, 2150-2156.

Lohmann, L.G. (2003). Phylogeny, classification, morphological diversification and biogeography of Bignonieae (Bignoniaceae, Lamiales) (PhD thesis). University of Missouri-St. Louis, St. Louis, MI.

Lohmann, L.G., Bell, C.D., Calio, M.F. \& Winkworth, R.C. (2013). Pattern and timing of biogeographical history in the Neotropical tribe Bignonieae (Bignoniaceae). Bot. J. Linn. Soc., 171, 154-170.

Maitner, B.S., Boyle, B., Casler, N., Condit, R., Donoghue, J., Duran, S.M., . . \& Enquist, B. J. (2018). The bien $\mathrm{r}$ package: A tool to access the Botanical Information and Ecology Network (BIEN) database.Methods Ecol. Evol ., 9 , 373-379.

Medina-Vega, J.A., Bongers, F., Schnitzer, S.A. \& Sterck, F. J. (2021). Lianas explore the forest canopy more effectively than trees under drier conditions. Func. Ecol. , $35,318-329$.

Meunier, F., Verbeeck, H., Cowdery, B., Schnitzer, S.A., Smith-Martin, C.M., Powers, J.S., Xu, X., Slot, M., De Deurwaerder, H.P., Detto, M. and Bonal, D. (2021). Unraveling the relative role of light and water competition between lianas and trees in tropical forests: A vegetation model analysis. J. Ecol. , 109, 519-540.

Meyer, L., Diniz-Filho, J.A.F., Lohmann, L.G., Hortal, J., Barreto, E., Rangel, T., et al. (2020). Canopy height explains species richness in the largest clade of Neotropical lianas. Glob. Ecol. Biogeogr. , 29, 26-37.

Moles, A.T., Ackerly, D.D., Webb, C.O., Tweddle, J.C., Dickie, J.B. \& Westoby, M. (2005). A brief history of seed size. Science, 307, 576-80.

Pausas, J.G. \& Austin, M.P. (2001). Patterns of plant species richness in relation to different environments: An appraisal. J. Veg. Sci. , 12, 153-166. https ://doi.org/10.2307/3236601

Powers, J. S. (2015). Reciprocal interactions between lianas and forest soil. In Ecology of lianas, ed(s). S. A. Schnitzer, F. Bongers, R. J. Burnham, \& F. E. Putz. Wiley Blackwell, Oxford, UK, pp. 175-187.

Putz, F.E. (1984). The natural history of lianas on Barro Colorado Island, Panama. Ecology , 65, 1713-1724.

Putz, F. E. \& Chai, P. (1987). Ecological studies of lianas in Lambir national park, Sarawak, Malaysia. J. Ecol. , 75, 523-531.

Putz, F.E. \& Holbrook, N.M. (1991). Biomechanical studies of vines. In: The Biology of Vines, ed(s). Putz, F.E. \& Mooney, H.A. Cambridge University Press, Cambridge, United Kingdom, pp. 73-97.

Reich, P.B. (2014). The world-wide 'fast-slow' plant economics spectrum: a traits manifesto. J. Ecol. , 102, $275-301$.

Rowe, N. \& Speck, T. (2004). Plant growth forms: an ecological and evolutionary perspective. New Phytol. , 166, 61-72.

Schnitzer, S.A. (2005). A mechanistic explanation for global patterns of liana abundance and distribution. Am. Nat. , 166, 262-76. 
Schnitzer, S.A. (2018). Testing ecological theory with lianas. New Phytol. , 220, 366-380.

Schnitzer, S.A. \& Bongers, F. (2002). The ecology of lianas and their role in forests. Trends Eco. Evol. , $17,223-230$.

Schnitzer, S.A. \& Bongers, F. (2011). Increasing liana abundance and biomass in tropical forests: emerging patterns and putative mechanisms.Ecol. Lett. , 14, 397-406.

Schnitzer, S.A., van der Heijden, G.M. \& Powers, J.S. (2016). Reply to Verbeeck and Kearsley: Addressing the challenges of including lianas in global vegetation models. Proc. Natl Acad. Sci., 113, E5-E6.

Schnitzer, S.A., Carson, W.P. \& Letters, E. (2010). Lianas suppress tree regeneration and diversity in treefall gaps. Ecol. Lett. , 13, 849-57.

Schnitzer, S.A., Dalling, J.W. \& Carson, W.P. (2000). The impact of lianas on tree regeneration in tropical forest canopy gaps: evidence for an alternative pathway of gap-phase regeneration. J. Ecol. , 88, 655-666.

Simard, M., Pinto, N., Fisher, J. B. \& Baccini, A. (2011). Mapping forest canopy height globally with spaceborne lidar. Journal of Geophysical Research, 116, G04021. https ://doi.org/10.1029/2011J G001708

Speck, T. \& Burgert, I. (2011). Plant Stems: Functional Design and Mechanics. Annu. Rev. Mater. Res. , 41, 169-193.

Sperotto, P., Acevedo-Rodriguez, P., Vasconcelos, T.N.C. \& Roque, N. (2020). Towards a standardization of terminology of the climbing habit in plants. Bot. Rev. , 86, 180-210.

Stevens, G.C. (1987). Lianas as structural parasites: the Bursera simaruba example. Ecology 68: 77-81.

Ustin, S.L. \& Gamon, J.A. (2010). Remote sensing of plant functional types. New Phytol. , 186, 795-816.

van der Heijden, G.M.F. \& Phillips, O.L. (2008). What controls liana success in Neotropical forests? Glob. Ecol. Biogeogr. , 17, 372-383.

van der Heijden, G.M.F. \& Phillips, O.L. (2009). Liana infestation impacts tree growth in a lowland tropical moist. Biogeosciences , 6, 2217-2226.

Vaughn, K.C. \& Bowling, A.J. (2011). Biology and physiology of vines.Hortic. Rev. (Am. Soc. Hortic. Sci.) , 38, 1-21.

Verbeeck, H. \& Kearsley, E. (2016). The importance of including lianas in global vegetation models. Proc. Natl Acad. Sci. , 113, E4-E4.

Verheijen, L.M., Aerts, R., Bonisch, G., Kattge, J. \& Van Bodegom, P.M. (2016). Variation in trait tradeoffs allows differentiation among predefined plant functional types: Implications for predictive ecology. New Phytol. , 209, 563-575.

Westoby, M., Falster, D.S., Molest, A.T., Vesk, P.A. \& Wright, I. J. (2002). Plant ecological strategies: Some leading dimensions of variation between species. Annu. Rev. Ecol. Syst. , 33, 125-159.

Wright, I.J., Reich, P.B., Cornelissen, J.H.C., Falster, D.S., Garnier, E., Hikosaka, K., et al. (2005). Assessing the generality of global leaf trait relationships. New Phytol. , 166, 485-496.

Wright, I.J., Reich, P.B., Westoby, M., Ackerly, D.D., Baruch, Z., Bongers, F., et al. (2004). The worldwide leaf economics spectrum. Nature, 12, 821-827.

Wyka, T.P., Oleksyn, J., Karolewski, P. \& Schnitzer, S.A. (2013). Phenotypic correlates of the lianescent growth form: a review.Ann. Bot. , 112, 1667-81.

Yorke, S.R., Schnitzer, S.A., Mascaro, J., Letcher, S.G. \& Carson, W.P. (2013). Increasing liana abundance and basal area in a tropical forest: The contribution of long-distance clonal colonization,Biotropica , 45, $317-324$. 


\section{Figures and Tables Legends}

Figure 1 - Framework used in this study. Liana species (woody climbers) may be classified into two main types of climbing on supports: passive and active mechanisms. Each group has different types of strategies (see explanation in Box 1). The upper blue arrow indicates an increase in complexity of the type of climbing mechanism from scrambler to tendril. The lower blue arrow indicates an increase in the maximum size (diameter) of the support to which each type of climbing mechanism can attach. Note that the constraint in size support is valid only for species with active climbing since species with passive climbing do not climb and pass over different supports.

Figure 2 - Functional trait differences among liana species per climbing mechanism type (a-f) based on synthesis from literature. Note that leaf area (d) and seed mass (f) are in the logarithmic scale.

Figure 3 - Results from the piecewise structural equation models for abundance of active and passive climbing species across the tropics. In (a), structural equation model explaining liana density per plot of the active climbing species in relation to forest structure, climate and soils. In (b), structural equation model explaining liana density per plot of the passive climbing species in relation to forest structure, climate and soils. TEMP $=$ temperature; $\mathrm{PREC}=$ precipitation; and $\mathrm{CEC}=$ cation exchange. Transparent arrows represent non-significant coefficients and dashed arrows represent marginally significant coefficients $(0.05<$ $P<0.1)$. Density is the number of individuals per ha. The inset map shows the location of the plots used in this study.

Figure 4 - Results from the piecewise structural equation models for richness of active and passive climbing species across the tropics. In (a), structural equation model explaining liana species richness per plot of the active climbing species in relation to forest structure, climate and soils. In (b), structural equation model explaining liana species richness per plot of the passive climbing species in relation to forest structure, climate and soils. TEMP $=$ temperature PREC $=$ precipitation; and $\mathrm{CEC}=$ cation exchange. Transparent arrows represent non-significant coefficients and dashed arrows represent marginally significant coefficients $(0.05<P<0.1)$. Species richness is the number of species per ha. The inset map shows the location of the plots used in this study.

Figure 5 - Map showing the relative proportion of species richness per climbing mechanism across biogeographical realms. Note that the relative proportion of active to passive climbing species richness is significantly higher in the Neotropics (Table S1). 


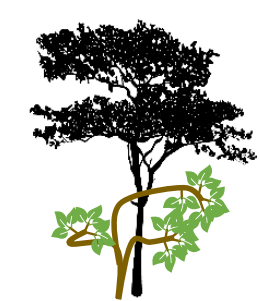

Passive Climbing

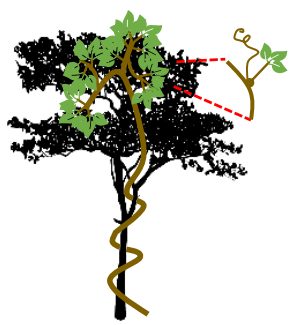

Active Climbing

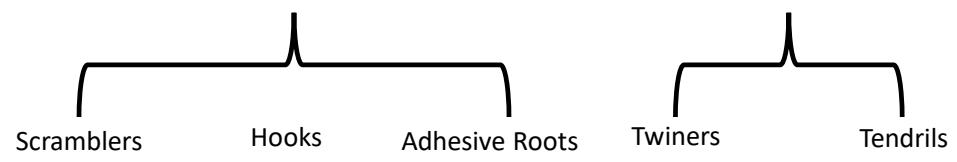

Complexity in climbing strategy

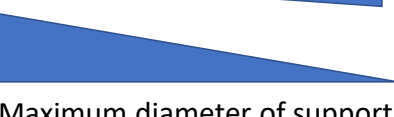

Maximum diameter of support
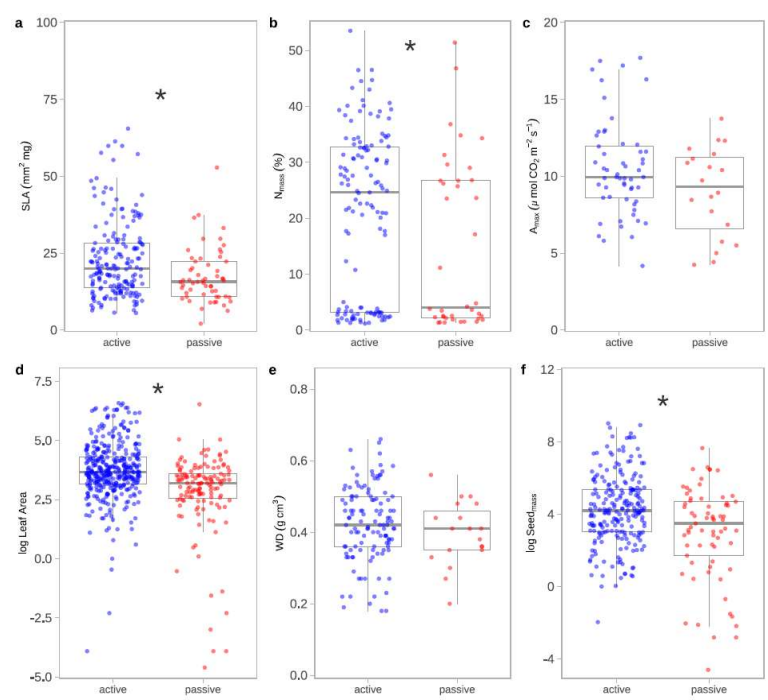

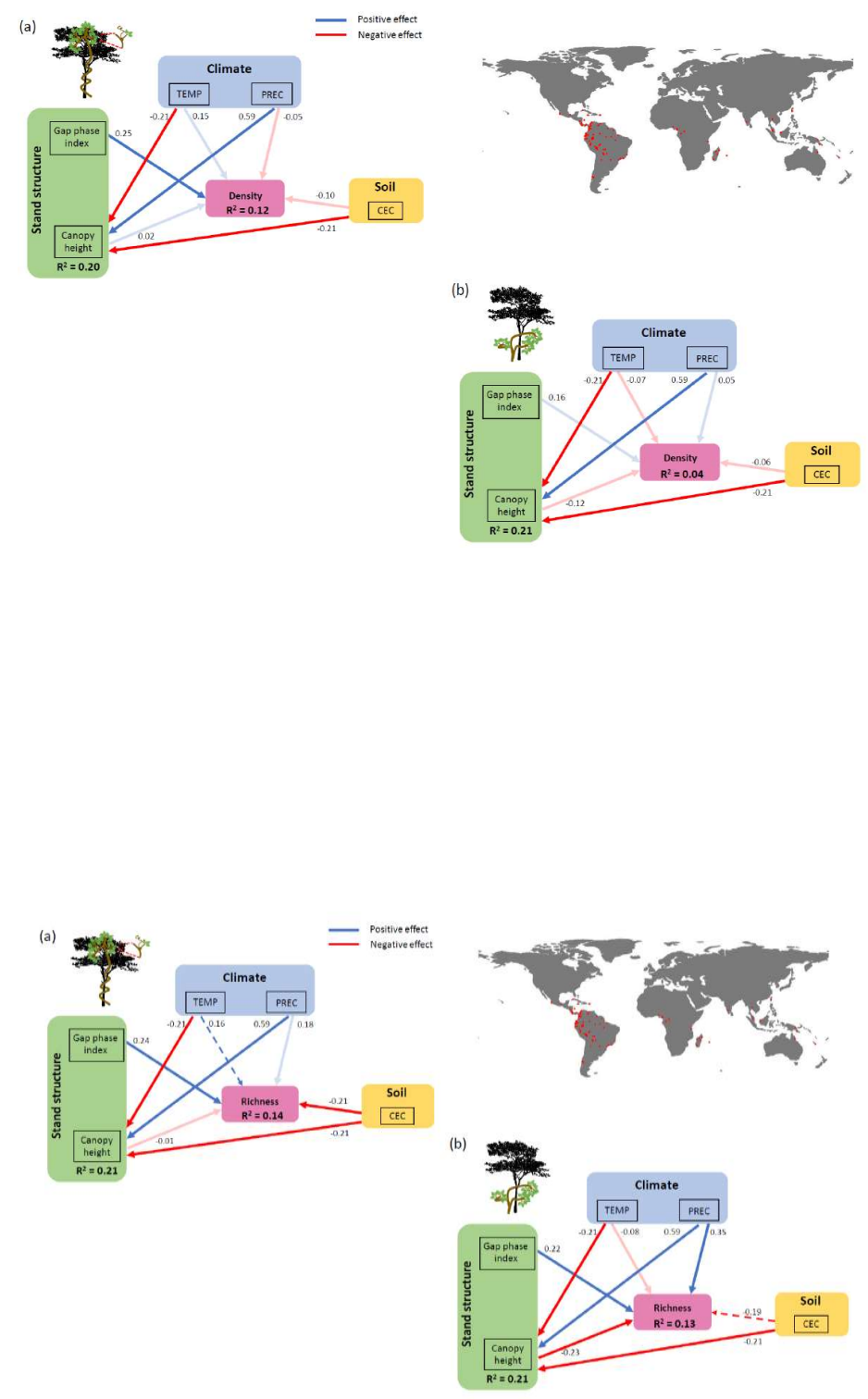


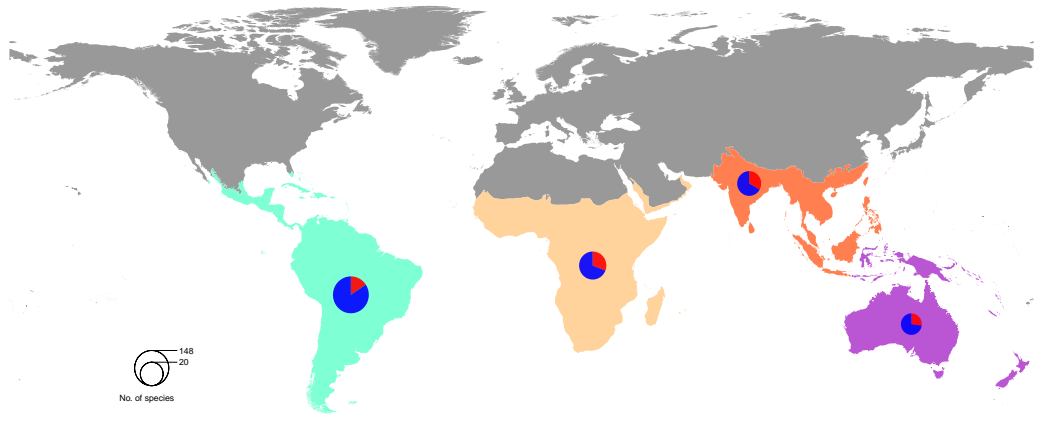

Climbing mechanism Passive Active 